\title{
Analysing communication dynamics at the transaction level: the case of Air France Flight 447
}

\author{
Lida Z. David' ${ }^{1}$ (D) Jan Maarten Schraagen ${ }^{1,2}$
}

Received: 26 December 2017 / Accepted: 12 July 2018 / Published online: 24 July 2018

(c) The Author(s) 2018

\begin{abstract}
A system's continuous adaptability is a vital determinant of its safety. It is thus very important for a system to reach graceful extensibility, the ability to adapt in unexpected situations (Woods in Reliab Eng Syst Saf, https://doi.org/10.1016/j. ress.2015.03.018, 2015). Current methods to study patterns of adaptation have mostly focused on relatively static network relationships of short time scales. We argue that both adaptive and maladaptive patterns of adaptation are rooted in patterned behaviours that should be studied in light of their previous history of transactions. Those patterns may develop over longer time scales yet exert their effects during unexpected situations on shorter time scales. In this study, we focused on communication patterns that played out during the Air France 447 incident. Butts (2008) relational event model was employed to examine the communication dynamics amongst the pilots in the cockpit of flight AF447, and illustrate how communication patterns may be studied by considering sequences of relational events, thus adopting a dynamic, de-contextualised approach to system analysis, at a 'transaction level'. The analysis of the communication transcript revealed patterned changes in some communication dynamics in the cockpit after the flight entered an unexpected situation, which led to the biased strengthening or weakening of certain links in the network. These changes-even though preliminary due to the limited number of agents analysed-suggest that capturing the structural composition of a system at the transaction level assists in explaining how transactions fail, and can be used for the development of better system structures or training procedures for system interaction.
\end{abstract}

Keywords Complex sociotechnical systems · Adaptability · Resilience · Transactions · Relational event model $\cdot$ Air France $447 \cdot$ Team communication

\section{Introduction}

The scientific community concerned with complex sociotechnical systems strives for maintenance of sustained adaptability, since the continuous ability of a system to adapt determines its safety (Woods 2015). However, sustaining adaptability in various dynamically changing environments poses great challenges. All systems have boundaries (Farjoun and Starbuck 2007; Hoffman and Woods 2011) that, if exceeded, render them unable to adjust to any new demands accordingly, and thus cannot preserve their adaptability. There have been many resilient-robust systems, where

Lida Z. David

1.david@utwente.nl

1 Department of Cognitive Psychology and Ergonomics, University of Twente, Enschede, The Netherlands

2 Netherlands Organisation for Applied Scientific Research TNO, Soesterberg, The Netherlands extensive modelling of various possible events allows for the identification of boundary locations and prepare systems to adjust depending on the event they face (Woods 2015). Still, systems continue to face the paradox of being 'almost totally safe' (Amalberti 2001; Reason 2000), since unexpected situations that are not modelled can always arise, precluding systems from adapting.

The direct relation between system safety and graceful extensibility, that is, the ability to adapt in unexpected situations (Woods 2015), makes it important to study and develop systems in such a way that there will no longer be a gap between the real world and the simulated one in which systems are developed and tested ('Doyle's Catch'; Woods 2016). For example, studying a system's underlying architecture may lead to improvement of the system's ability for sustained adaptability independent of the event it might face, by improving its foundations' capacity for adjustment, instead of focusing on identifying superficial, content-dependent boundaries (Doyle and Csete 2011; Schraagen 2017). The 
present article aims to examine a novel approach to the analysis of systems' architectures that moves beyond contextualised boundaries. In the following sections, we explore this approach and how it deviates from, or adds value to already existing architectures, followed by an exploration of the statistical analysis that could couple the approach under consideration.

The ideas and concepts of this article are founded on systems theory, which takes a hierarchical view to system structure where different architectures can exist at different levels in the hierarchy (Newell 1982). Following the systems theory approach, multiple architectures for adaptability exist; primarily at the cognitive, lower system level (e.g., ACT-R; Anderson 2007) that focuses on the individual, and the higher, knowledge level (e.g., SOAR; Newell 1990) also known as the 'rational band', which addresses shared knowledge across individuals. However, these architectures do not easily scale up to complex sociotechnical systems. Partially as a response, the concept of 'macrocognition' was developed (Klein et al. 2003; Schraagen et al. 2008), incorporating the study of cognitive adaptations to complexity (Schraagen et al. 2008). It distinguishes sense-making, planning, adaptation, problem detection, and coordination as important macrocognitive functions, which are not only performed by individuals or teams, but also organizations, or joint cognitive systems that coordinate people with technology. Other approaches take a still broader view on complex sociotechnical systems, incorporating system constraints [Cognitive Work Analysis (CWA); Vicente 1999], control structures [Systems Theoretic Accident Model and Processes (STAMP); Leveson 2004], task, social, and information networks [Event Analysis of Systemic Teamwork (EAST); Stanton et al. 2005, 2008, 2013), or performance variability and resonance [Functional Resonance Analysis Method (FRAM); Hollnagel 2012]. These approaches have been used for risk assessment (e.g., Stanton and Harvey 2017), analysing accidents (Allison et al. 2017; Griffin et al. 2010), or system design (Stanton et al. 2016).

Macrocognition is distinguished from microcognition primarily by its time scale of analysis. Whereas the latter focuses on cognitive processes in the time band of $100 \mathrm{~ms}$ up to $10 \mathrm{~s}$, the former focuses on cognitive processes from minutes to hours. The preferred means by which these processes are studied then also varies, with microcognition frequently opting for constrained tasks in confined environments with high experimental control, while macrocognition opting for real-life tasks under actual working conditions with less experimental control (Cacciabue and Hollnagel 1995; see; Hoffman and McNeese 2009 for a historical overview).

Interestingly, although in principle macrocognition extends to the organizational level, in practice there are very few studies employed by organizational scientists that implement such methods. Also, the time scales adopted in macrocognitive studies are not weeks, months or years, but rather a few hours at most, focusing on a specific time and place where systems failed to adapt. Methodologically, then, an important shortcoming in current macrocognitive studies is the lack of de-contextualised longitudinal data collection, which prohibits the discovery of emergent behaviours at different or longer time scales.

\subsection{The transaction level}

We propose that there exists yet another system level, which we call the 'transaction level', developed to enable the analysis of systems under diverging, prolonged time scales. The transaction level comprises a 'true system level', as this is defined by Newell (1982). In Newell's sense, a system level is a reflection of the nature of the physical world, not simply a level of abstraction. Each level within the hierarchy of systems levels comprises an aggregation of characteristics present at lower levels, along with an addition of meaning. In turn, this meaning leads to some emergent system properties, which come to define the system at hand, concealing the now invisible lower-level properties. Therefore, although the levels are ontologically irreducible, each level may still be implemented at the next lower level.

In contrast to the knowledge level, the concept of 'goal' does not play a role at the transaction level. Instead of focusing on the context of knowledge exchanged between agents in a system and the static form in which this is presented, the focus turns to the strength and reciprocity of the transactions made between agents, and how these dynamically change over time. To better understand the added value of the transaction level and its difference to other system levels, a quick overview of its components, its laws of composition, its medium, and its behaviour laws, is in order.

The system at the transaction level, the entity to be described, is the network. The system's primitive elements, the components that form the basic network structure, are nodes and links. Nodes refer to a set of agents, either human or technical, and links refer to the possible connections between those agents. Those components are assembled into systems by laws of composition that yield nodes and links with varying strength and reciprocity. The medium at the transaction level is the transaction (as might be suspected), and transactions are generated through the links that are formed between nodes. The transactional content may differ widely, from affect and influence to goods and services, and information. Finally, the law of behaviour, i.e., how the system depends upon its components and composition, is the principle of 'relationality': links are selected to attain transactions. As links are characterized by strength and reciprocity, the generation of transactions is dependent on these notions; all of which can change from one situation to another. For example, different situations might lead to 
the formation of different 'hubs' in the network, referring to 'highly connected nodes' that reflect different transaction patterns (Berlingerio et al. 2011, p. 1).

Examining the possible patterns of change in transactions when a system enters an unexpected situation can be vital for understanding how its architecture changes, and how transaction patterns that were successful during a known situation may weaken or change during a surprise one, contributing to failure.

It is particularly important to understand that the transaction level depends on the principle of 'relationality', thus considering the structural strength and diversity of connections between nodes and how those unfold, instead of focusing on the 'rational', contextualised knowledge those nodes exchange. This enables the development of analyses that not only offer de-contextualised insights, but can also allow scientists to understand and define a system through the collection and consideration of longitudinal data.

It should, however, be mentioned that the transaction level is a radical approximation, and thus may be poor in predicting some ranges of behaviour, as for example, when team members do not know each other well and have not built up structural links with each other. Hence, considering that all system levels play a role simultaneously at all times, invoking the knowledge level could provide insights that the transaction level is unable to offer. In this example, invocation of the knowledge level would be the only way to explain absent, inappropriate, incomplete or misunderstood transactions. Consulting the knowledge level is also related to the possible restrictions on communication bandwidth. Even if individual team members possess all relevant knowledge, they may be unable to share all that knowledge, particularly in stressful situations, due to the fact that they can only speak, see and hear so much, thus narrowing down their communication bandwidth. A team of experts is not by definition an expert team, if team members do not know when to communicate what information to whom, or are afraid to speak up. While the transaction level could determine the restrictions to the system's bandwidth, invoking the knowledge level could give more insights to the nature of the communication patterns.

The emergent properties of the transaction level add value to existing literature around sociotechnical system analyses, as this will be further elaborated upon in the following section, through considering how our approach relates to alternative approaches.

\subsection{The transaction level versus alternative approaches}

The emphasis on the exchange of transactions between actors in a network is very similar to what Stanton et al. (2006) described in their theory of distributed situation awareness (see also Stanton et al. 2009; Sorensen and Stanton 2015). These authors took a systems' level approach and noted that what mattered was that the right information was passed to the right agent at the right time, instead of all information being available to a single human agent. Neville et al. (2016) also used the concept of 'transaction' in a similar, though more restricted, way than we do. They referred to transactions as an exchange of situation awareness between agents rather than a mere communication between them. According to Neville et al. (2016), transactions are enriched by specific and individual interpretations of each agent and so may provide a clue to other agents as to what one individual is working on. Since transactions hold the key to safe and efficient performance, accident investigators need to understand not only what information was lost, but also what transactions were inadequate or were required but not forthcoming (Salmon et al. 2016; Stanton and Harvey 2017).

Although quite similar in spirit, Stanton et al.'s approach differs from our description of the transaction level in several ways. First, while Stanton et al. describe situation awareness as a system level phenomenon rather than an individual level phenomenon, their concept of a 'system level' differs from Newell's proposed concept. Juxtaposing individual levels and system levels amounts to an aggregation of units, moving from the individual to the team or organisation, or 'system' (cf. Karsh et al. 2014; Hackman 2003). This makes 'levels' similar to 'perspectives' or 'levels of abstraction', whereas in Newell's view system levels are, as mentioned earlier, a reflection of the nature of the physical world, describing phenomena that emerge from their components (Hackman 2003).

Second, Salmon et al. (2016), in the application of their Distributed Situation Awareness (DSA) theory to the Air France 447 accident, focused on the sharp end of the communication patterns of the pilots involved. Again, this is a knowledge level analysis rather than a transaction level analysis. What needs to be determined, if we are looking for architectures for sustained adaptability, is whether underlying, structural relational patterns that were present in the cockpit led to any specific exchange of information at any particular moment in time.

In agreement with Salmon, Walker and Stanton (2016), the transaction level supports the notion that failed transactions lie at the root of accidents occurring in complex sociotechnical systems. However, this statement alone does not explain how transactions fail. This can only be understood by considering an analysis at the transaction level: first, by determining structural differences in links' strength and reciprocity; second, by identifying restrictions on communication bandwidth. As to the first aspect, one needs to ask whether team members have been able to develop links to each other, and what the power relations between those links are. This is related to the second aspect, since structural 
relations can help in determining the restrictions in the network's communication bandwidth. Through these steps, one recognizes the network structure upon which transactions occur, and can thus identify the nature of transaction failures (see also Stanton and Harvey 2017).

Recently, Roth (2018) applied what he called a 'transactional approach' to an analysis of the crash of TransAsia Flight GE235. Roth (2018; see also Roth and Jornet 2013) distinguishes three approaches to modelling cognition: selfaction, interaction, and transaction. The self-action perspective explains human behaviour in terms of goals, plans and other internal representations. Interactional approaches are closely aligned with joint cognitive systems and DSA perspectives, focusing on the information shared between any two agents. Although interactions cannot be reduced to individual agents, agents are still modelled independently and are said to exchange situation awareness. Transactional approaches emphasize the unity/ identity of organism and environment, constituting a single, irreducible system. Although superficially similar, a 'transactional approach' as described by Roth (2018) should not be equated with the transaction level. As noted above, approaches to describing organism and environment are not the same as system levels that describe phenomena that emerge from their components. Hence, we do not deny the importance of the cognitive and knowledge levels, nor of any of the other lower and, possibly, higher, levels. Cognitive constructs such as mental representations, as well as knowledge level constructs such as goals and knowledge are valuable in their own right in explaining particular phenomena, usually associated with particular time scales at which these phenomena occur (Newell 1990). What we do claim, by introducing the transaction level, is the importance of concepts such as nodes, links and transactions in explaining certain behaviours. These concepts are not by any means new, and indeed have been used extensively in the network sciences (e.g., Alba 1982; Wasserman and Faust 1994), as well as in methods such as EAST (Stanton et al. 2005, 2008, 2013). What we hypothesize by introducing the transaction level, is the existence of patterned behaviours that can only be studied by taking the previous history of transactions into account. Therefore, we need to go beyond static descriptions of social networks and take sequences of relational evens into account.

\subsection{The relational event framework}

Recent conceptualizations (e.g., Cooke et al. 2013) view team cognition as a context-dependent team interaction, rather than a monolithic entity that a team can either have or not have. In other words, rather than viewing team cognition as something that is shared among team members and then aggregated, it is viewed as an interdependent network that should be studied at the team level. Within such networks, the relations between actors, and the maintenance of these relations throughout time, are necessary for successful team operation (Johansson and Hollnagel 2007). Similarly, Leenders et al. (2016, p. 97) argue that "we need to imbue our theories and analyses of team process with more temporal constructs". They propose the relational event as an appropriate unit of analysis, which refers to a sender initiating an action towards a target. By performing an analysis in a 'sequence of relational events', the model enables the investigation of transactions between nodes while also considering their past transactions. We propose that Butts' (2008) relational event framework, a statistical way of analysing sequences of relational events, is appropriate for system analysis at the transaction level. It can capture transaction patterns across different nodes and extended time scales considering past and current transactions. Furthermore, relational event sequences can be investigated quantitatively, as can the tendency of the system to encourage or discourage some of them, without the need for specific content information.

The framework focuses on certain communication dynamics (discussed in more detail below) that can be investigated via the relational event model (REM), namely individual level heterogeneity, preferential attachment, cognitive effects, triadic effects, and communication norms. These dynamics may lead to patterned biases in transactions, and thus to the formation of certain hubs between some nodes. As these dynamics change, so do the hubs' patterns and strength within the network. Their patterns can reflect the underlying 'rules' driving the system to successfully adjust depending on the demands of the situation. However, changes in these dynamics could lead to the biased formation or strengthening of certain hubs that deprive the system of its capacity for successful adjustment. Information on each dynamic and its parameters is provided below, as described by Butts (2008).

\subsubsection{Individual level heterogeneity}

Individual level heterogeneity refers to unobserved heterogeneous endogenous or exogenous attributes of nodes, such as differences in context, training or institutional role that make an event between two nodes more likely to occur. In the REM, this is captured with 'fixed effects' for participation.

\subsubsection{Preferential attachment}

Preferential attachment (PA) is when a node that was contacted more in the past is more likely to be contacted by other nodes in the future, while new nodes prefer to attach to well-connected nodes over those less well-connected. In the REM, PA is considered present in the data when its associated parameter is positive. 


\subsubsection{Cognitive effects}

The cognitive processes of memory and perception might affect hub formation and lead to some events occurring more or less frequently than others. Such processes can lead to the appearance of Recency (R) effects, making nodes that have been contacted more recently by a sender more cognitively available to that sender, and thus more likely to be a future target (Snijders et al. 2006). They can also lead to Persistence (P) effects, also known as 'inertia' (Leenders et al. 2016), where one node is more likely to direct communication towards a target whom it had contacted more in the past, since those more frequently contacted are more available in memory (Romney and Faust 1982; Freeman et al. 1987). The presence of $R$ and $P$ is captured by a positive parameter, while a negative one suggests other mechanisms are at play.

\subsubsection{Conversational Norms}

Gipson (2003), presented a framework to study local rules in communication through participation shifts (hereafter referred to as 'P-shifts'), namely the moment-by-moment shift patterns of individuals between the role of sender and target. An example of a P-shift is reciprocity between two nodes (AB-BA). These $\mathrm{P}$-shifts reflect the underlying rules in any communication phenomenon that allow the system to generate meaningful structured experiences rather than chaotic episodes, as well as the different opportunities of nodes to be senders or receivers of transactions according to differences in attributes or situational demands (Gibson 2003). Their positive or negative parameters indicate the reliance on, or independence of nodes from them.

\subsubsection{Triadic effects}

Triadic effects refer to two-path effects, deriving from the notions of transitivity and cyclicity. More specifically, in a two-path communication one node contacts another node, which will in turn direct contact to someone else. This might affect the likelihood of the initial sender contacting the end target ('outbound two-path'; T-OTP), or of the end target contacting the initial sender ('inbound two-path'; T-ITP). Triadic effects also include shared partner effects. For example, two nodes that have contacted the same target in the past, are more likely to communicate with each other in the future ("outbound shared partner"; T-OSP), as do two nodes that have been contacted by the same node in the past ("inbound shared partner"; T-OSP). Their positive and negative parameters show whether the effects were encouraged or discouraged.

\subsection{Aim of the study}

We will use the Relational Event Model to assess the existence of each of the five communication dynamics within the system in the cockpit of flight 447 . This case is a prominent example of a complex system failure in aviation under an unexpected emergency situation. On 31 May 2009, the Air France flight 447 crashed into the Atlantic Ocean, and after extensive investigations it was concluded that the airplane had stalled and crashed as a result of poor flight inputs that followed the freezing of the Pitot tubes of the airplane. The freezing of the Pitot tubes caused the autopilot system to disconnect, placing the flight into an unforeseen situation, in which the system failed to adjust accordingly. Our analysis aims to show how the pilots' underlying communication architecture, as captured by communication dynamics within the cockpit, contributes to the explanation of transaction failures. Hence, one will be able to understand how this systems' level is ideal for analysis of prolonged time periods, since it captures the system's architecture considering prolonged periods of relational event sequences, in a contentindependent manner. It is worth noting that our analysis is based on a 2-h availability of the Cockpit Voice Recorder (CVR) transcript, but the REM captured communication patterns that could extend to even more prolonged normal flight or emergency flight periods.

The aforementioned five communication dynamics amongst the crew members in the cockpit of AF447 were assessed using the transcript of pilots' communication (BEA 2012), in an attempt to determine if the REM can capture changes in these dynamics when the system enters an unexpected situation, and whether this potential change could contribute to the explanation of how transactions fail.

\section{Method}

\subsection{Dataset}

The data used for the analysis was extracted from the official communication transcript of the AF447 flight, which was part of the investigation report provided by the French authority Bureau d'Enquêtes et d'Analyses pour la sécurité de l'aviation civile (BEA 2012). The transcript includes events such as conversation between the pilots, radiotelephonic messages and other sounds that occurred in the cockpit throughout the course of the flight. Our analysis considered only transactions between the human agents in the cockpit; the Captain (Marc Dubois), the Pilot in the right seat (Pierre-Cédric Bonin), and the Pilot in the left 
Table 1 Example rows of the coded transcript

\begin{tabular}{llll}
\hline Time & FromId & ToId & Phase \\
\hline $00: 31: 00.0$ & 1 & 2 & Normal \\
$00: 31: 00.1$ & 2 & 1 & Normal \\
$00: 31: 04.0$ & 2 & 1 & Normal \\
$00: 31: 15.0$ & 1 & 2 & Normal \\
$00: 31: 15.1$ & 2 & 1 & Normal \\
\hline
\end{tabular}

seat (David Robert). The Captain and co-pilots are hereafter respectively referred to as $\mathrm{CPN}, \mathrm{PF},{ }^{1}$ and $\mathrm{PNF}^{2}$

\subsection{Coding scheme}

A coding scheme was developed for the analysis of the transcript, coding each communication event as this unfolded over time. The coding was carried out by three human factors students, with a mean age of $24.7\left(\mathrm{SD}_{\text {age }} 2.51 ; 2\right.$ females, 1 male), who were pursuing their Master of Science degree in Human Factors and Engineering Psychology at the University of Twente in the Netherlands.

Using an excel document, four columns were generated; 'Time', 'FromId', 'ToId', and 'Phase'. The 'Time' column consisted of the exact time in which each relational event occurred, as indicated in the transcript (hh:mm:ss.ms). The 'FromId' column included the agent who was the sender at each particular event in time, while the 'ToId' included the target. Both columns consisted of coded numbers that represented different members of the crew. The CPN was coded as 1 , the PF as 2, and the PNF as 3. All relational events were considered in dyads, meaning that whenever one agent directed an action towards more than one target, data were coded as separate dyadic events with one written just below the other. To ensure the events were considered separately in the analysis but still not as two significantly different events, the second one was given a tiny offset (of $1 \mathrm{~ms})$. For example, when the CPN directed an action to both pilots, the action was coded as two individual events, both with Captain as sender, but each with a different target; $\mathrm{PF}$ and PNF. Any communication indicated by ' (...)' in the transcript (BEA 2012) was excluded, since it consisted of personal information irrelevant to the flight.

The 'Phase' column divided the data into two sets:

1. Normal phase. From the beginning of the flight, until the autopilot disconnection (00:31:00.0-02:08:12.0).

\footnotetext{
1 Pilot flying; Pierre-Cédric Bonin. The pilot who took the controls once the Captain left the cockpit.

2 Pilot not flying; David Robert. Co-pilot.
}

2. Emergency phase. From the autopilot disconnection to the end of the flight (02:08:19.0-02:14:26.9).

Table 1 presents an example of the first five rows of the coded transcript.

It should be noted that in the normal phase, only $10 \%$ of the events occurred when three agents were in the cockpit, with $90 \%$ occurring when only two were present. In the emergency phase, these percentages changed to 61 and $38 \%$, respectively.

To ensure inter-rater reliability, and since the coding process was rather simple, every analyst was given a set of rows to code that included half of the rows of the previous analyst. No mistakes were noted in the coded data.

\subsection{Analysis}

The coded file was inserted in the $\mathrm{R}$ environment for statistical computing (R Core Team 2013) and used for the estimation of the following five communication dynamics: Fixed effects, preferential attachment, triadic effects, persistence, recency, and participation shifts (p-shifts). The p-shifts modelled, as denoted by Gibson's (2003) initials, were reciprocation (AB-BA), "handing off" of communication (AB-BY), persistence of source or target (AB-AY, $\mathrm{AB}-\mathrm{XB})$, and source "attraction" ( $\mathrm{AB}-\mathrm{XA})$. The remaining p-shifts were not considered since the data involved only three agents. All models were estimated using the dedicated library from the relevent package (Butts 2008).

The data were treated in two separate sets, distinguishing the normal from the emergency phase, and the five communication dynamics were estimated twice, once for each. Beginning with the normal phase, the rem.dyad() function was used to create a null model, in which all effects were considered as equally probable to explain the data, and thus served as a baseline reference for comparison of other models. Afterwards, the same function was used to fit multiple relational event models to the data. The codes for the construction of each model were extracted from the relevent library (Butts 2008), and can be found in Appendix A. Each model yielded a Bayesian information criterion (BIC) score, which indicates the predictive value of models in a dataset and is frequently used in statistics for model selection amongst a finite set of models. Therefore, the BIC statistic from each fitted relational event model was used for an initial model pruning, by examining each model's goodness-of-fit. In other words, models were compared to the baseline to assess whether they were capable of predicting the data. Redundant models were disregarded. Then, parameter estimates along with their approximate $95 \%$ confidence intervals, obtained using maximum likelihood under the interval time model, were considered and plotted on a scatterplot 
Table 2 Data size and BIC statistics for the fitted relational event model

\begin{tabular}{lll}
\hline & \multicolumn{2}{l}{ BIC } \\
\cline { 2 - 3 } & Normal phase & Emergency phase \\
\hline$N$ & 3 & 3 \\
$M$ & 268 & 177 \\
Null & 3153.566 & 2220.569 \\
PS & 2658.967 & 1481.695 \\
R & 2706.800 & 1347.411 \\
P & 2884.386 & 1756.606 \\
PA & 3073.555 & 2125.571 \\
FE & 2863.169 & 2162.281 \\
T & 3168.540 & 2044.386 \\
PS +R & 2663.609 & 1191.209 \\
PS +R +P & 2647.294 & 1188.209 \\
PS +R +P+PA & 2652.586 & 1226.974 \\
\hline
\end{tabular}

via the geom_point() function of the package ggplot2 (Wickham 2009). Estimates were valuable for the interpretation of the data, since the positive or negative direction of each estimate reveals the respective encouragement or discouragement of the effects at each particular phase. The same analysis was followed for the emergency phase.

\section{Results}

Below follows a comparison of BIC scores for each fitted relational event model, as well as plotting and interpretation of parameter estimates of the effects. This section aims to explain the results from the analysis and offers an initial explanation of the findings, whose insights and added value in understanding sociotechnical systems will be further explained and expanded upon in the "Discussion" section. To facilitate analyses intended to further investigate the present results, or extend the scope of the present article, a detailed table with the exact parameter estimates, standard error, $z$ values and $p$ values, is provided in Appendix B.

Before exploring the results of the analysis, it is important to consider that many of the communication dynamics analysed, such as triadic effects or p-shifts, require the presence of a minimum of three nodes in the system. This was the case for only $10 \%$ of the data in the normal phase and $61 \%$ in the emergency. However, analysis was still possible due to the nature of the relational event model (REM) and its ability to consider preceding communication patterns. Therefore, the present results provide some fundamental insights in how communication dynamics change in unexpected situations, but readers should proceed with caution in drawing definite conclusions, as estimates may not be as precise.

\subsection{Model pruning}

Data size and the BIC scores for each model were gathered and presented in Table 2, which consists of three blocks. The first block (rows $N$ and $M$ ) includes the descriptive statistics for the size of the data at each phase. $N$ refers to the number of agents, and $M$ refers to the total number of events captured. The second block consists of the BIC scores for the fitted relational event models. The null model is fitted and presented at the null row, and its BIC score is used as baseline for comparison of the fitted models of the respective dataset. Each following model matches its corresponding effect, coded using the effect's initials: p-shifts (PS), recency $(\mathrm{R})$, persistence $(\mathrm{P})$, preferential attachment (PA), fixed effects (FE) and triadic effects (T). Since only one effect is presented at each row, values can be considered as evidence for marginal effects, indicating the likelihood of this model to fit the data if all other models are kept constant. The third block contains the possible combinations of effects, allowing the investigation of better model-fit while considering different hub formations.

Before examining the table, it is necessary to know that a BIC score lower than the baseline implies that its corresponding model is preferred over the null model; i.e., a model whose BIC value is lower than the baseline, fits the data better and is thus more predictive of the communication dynamics governing the cockpit at a particular phase.

Inspecting the normal phase, four models seem to fit the data better than the null model: p-shifts (PS), recency (R), persistence (P), and preferential attachment (PA). Of these, the scores of PS and R do not deviate a lot from each other, indicating that both can offer a similarly good explanation to the data. While models for P and PA are closer to the null and are thus not as predictive of the data, they should not be disregarded; their addition to the model combination $\mathrm{PS}+\mathrm{R}+\mathrm{P}+\mathrm{PA}$ offers a BIC score smaller than $\mathrm{PS}+\mathrm{R}$ alone, depicting the added value of the two models in predicting the dynamics in the dataset. The model for triadic effects (T) does not enter the BIC optimal model, and is thus redundant to the analysis of this dataset. The effect of fixed effects is not considered here, since it has a non-significant $z$ value.

For the emergency phase, a quite similar pattern arises, indicating that the same models are predictive of the communication dynamics. However, it is worth mentioning that there is an increase in the predictive power of recency $(R)$ and persistence $(\mathrm{P})$, since the former now has the strongest marginal effects, while the latter fits the data better than it did in the normal phase. Also, the model for triadic effects (T), even though rather close to the baseline, enters the BIC optimal model, and could thus be useful in the interpretation of the dynamics. The changes in the predictive values of models across the two phases may be attributed to the 
Fig. 1 Parameter estimates and approximate $95 \%$ confidence intervals for the normal and emergency phases, MLE models

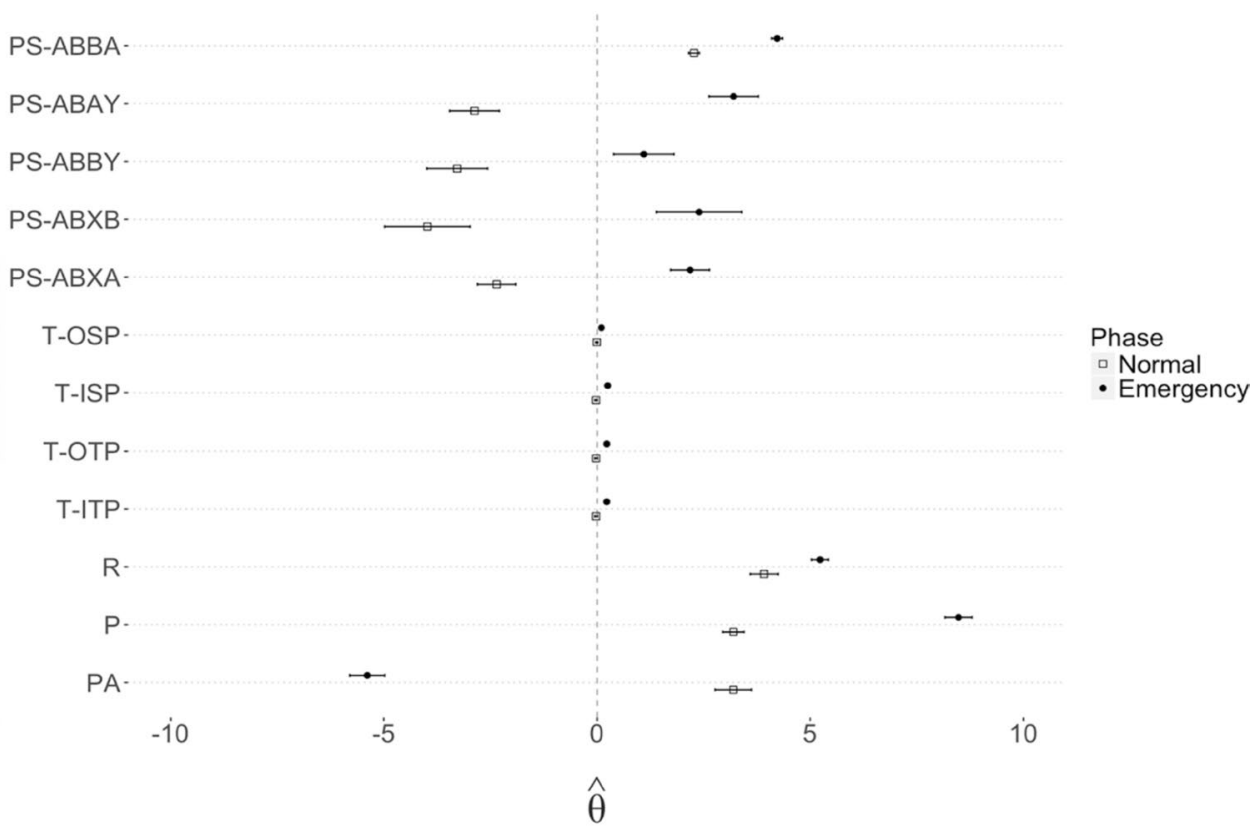

increased portion of relational events occurring when more agents were present in the cockpit. In the emergency phase, three agents were present for a larger number of events than in the normal phase, therefore allowing more triadic or other complex effects to occur.

\subsection{Parameter estimates}

While BIC values offer a first suggestion as to which models can predict communication dynamics at each phase, more precise conclusions are needed to recognize the exact changes in these dynamics throughout the course of the flight. Inspection of the effects' parameter estimates can reveal the direction of the effects in each phase, i.e., their respective encouragement or discouragement. Those, along with their approximate $95 \%$ confidence intervals, are plotted for both phases in Fig. 1, in the following order: P-shifts (PSABBA, PS-ABBY, PS-ABXA, PS-ABXB, and PS-ABAY), triadic effects (T-ISP, T-OSP, T-ITP, T-OTP) recency (R), persistence $(\mathrm{P})$, and preferential attachment $(\mathrm{PA})$. The effect of fixed effects was calculated but is excluded from the plot, since it is not a single-parameter effect and thus cannot be plotted as one.

From Fig. 1, and consistent with the inspection of the $\mathrm{BIC}$ values, it is clear that p-shifts, recency, persistence, and preferential attachment have strong marginal effects, while triadic effects are weak. The reversal in the value of p-shifts' estimates from negative in the normal phase to positive in the emergency phase, suggests that local rules were discouraged in the former but encouraged in the latter. This highlights the increased dependency on local rules when the system was faced with an emergency situation, implying that pilots, contrary to what they did under normal flight, now disregarded prior communication patterns that were based on institutionalized procedures or prior communication arrangements, and focused on non-standardized, local patterns of communication.

In addition, one can acquire further details on the changes in the prevalence of local rules across the two phases by examining specific p-shift patterns and how these change. For example, from Fig. 1 we see that persistence of source (PS-ABAY) may be the root of more malfunctioning than handing-off communication (PS-ABBY), since the estimates of the former are more positive than the latter. However, standard error (as indicated by the error bars in Fig. 1) is rather strong for $\mathrm{p}$-shifts in the present analysis. High uncertainty could be attributed to the presence of only two or three agents in the cockpit, hence more nodes are needed for more certain results. It is also interesting to note that reciprocity (PS-ABBA) is positive in both phases, while other p-shifts were only present in the emergency phase. This implies that communication dynamics depended on reciprocity throughout the entire flight, whilst other p-shifts were encouraged only during the unexpected situation. The positive value of reciprocity in both phases may, however, be due the majority of events in the normal phase occurring in the presence of only two agents, thus leaving little room for non-reciprocal events.

Inspecting the recency $(R)$ and persistence $(P)$ parameters, one can see that those remained positive in both phases, but became stronger during the emergency, placing greater dependency on cognitive factors such as short-term memory. This reflects more social inertia when faced with an emergency situation, since relational events were more reliant on 
the fraction of prior occurrences of the same relational event than in the normal phase. In other words, pilots were more likely to direct communication to those more cognitively readily available during the emergency.

The increased promotion of local rules and cognitive effects highlight the decreased dependency on institutionally based trained patterns of communication, which is also evident in the dramatic change in the preferential attachment (PA) of the pilots' communication pattern. While preferential targeting of nodes with higher prior communication was present in the normal situation, this changed in the emergency phase, where there was no trace of the preferential attachment effect explaining the data. This implies that during the emergency, the already existing links between nodes, based on status of institutional role or trained procedures, were suppressed, and new connections were formed between nodes that were not previously linked. Simply stated, pilots seem to have established their communication following endogenous mechanisms, trying to reach pilots whom they did not necessarily have had more interactions with in the past.

Triadic effects $(\mathrm{T})$ were disregarded as important communication dynamics, since their parameter estimates were weak, and only a small proportion generated significant $z$ values; an effect that could be attributed to the limited number of nodes analysed.

\section{Discussion}

This paper aimed to demonstrate how an analysis at the transaction level can be performed using the relational event model (REM), thus generating a systems level approach that could be applied to natural environments of diverging context and prolonged time scales. We examined whether the communication dynamics in the cockpit of AF447, and their pattern of change after the system entered an unexpected emergency situation, assisted in explaining the transaction failures in the system's network. The findings showed that there were indeed changes in the communication dynamics that governed each flight phase, especially in the conversational norms and the cognitive effects ruling the cockpit. Both became significantly more prominent once the flight entered the emergency phase, the same phase in which previous research has suggested that transaction failures in the cockpit escalate (Salmon et al. 2016). The correlation between the structural differences that occurred in the cockpit, and the increased inappropriate, incomplete, or missing transactions discussed by Salmon et al. (2016), is a first indication that the nature of these transaction failures is rooted within the system's architecture. The structural changes in links' strength and reciprocity across phases underlie the ultimately restricted communication bandwidth in the network. In other words, these changes refer to the increased reliance of the pilots on immediately preceding communication patterns during the emergency, rather than on pre-meditated, conscious decisions for distribution of communication; a transition that might underlie the impaired communication between the pilots when encountered with an unexpected situation. Our finding that pilots increasingly responded to immediately preceding local communication events also resonates with the BEA's finding that " $[t]$ he loss of coordination and the willing but chaotic cooperation in managing the surprise generated by the autopilot disconnection led quickly to the loss of cognitive control of the situation, and subsequently to the loss of physical control of the aeroplane." (BEA 2012, p. 184).

The patterned changes in the underlying dynamics can contribute to the explanation of some transaction failures by altering the power relationships between the pilots, therefore forming a dysfunctional network architecture [which the BEA report refers to as the 'inversion of the normal hierarchical structure in the cockpit' (BEA 2012, p. 185)]. Since some changes in dynamics between the pilots were prominent, it is logical to assume that dynamics between other nodes in the system underwent changes as well, thus underlying transaction failures in the whole network. However, it should be noted that since the analysis of the present article focuses on only three agents, whose presence was proportionally different between phases, conclusions should be drawn with caution. Therefore, after an initial exploration of the current findings and possible implications, the discussion will focus on comparing our approach to others, and exploring the feasibility and implications of using the REM for analysing systems at a transaction level.

The analysis of communication patterns revealed a critical change in communication, which implied that the rules governing the system when its environment had 'high validity'-referring to the relative stability between the cues provided by the environment and the outcomes of possible actions-changed when its validity became low, due to an ill-structured, uncertain, and fast-changing environment (Kahneman and Klein 2009). Even though a change from standardized communication patterns to chaotic information exchange may be expected under unforeseen situations, our findings move beyond this mere depiction of change. Indeed, an initial observation of the results suggests that pilots abandoned standardized procedures of communication when faced with the emergency, and engaged in more locally based conversation norms. Investigating differences more thoroughly, an increase in auto-correlations between local communication events was noted, meaning that the communication between the pilots was determined by immediately preceding events; an auto-correlation is generally thought to indicate imminent critical transitions and decreased resilience (Scheffer 2009). The pilots also relied on their memory 
and immediate perception, which made some agents more cognitively available than others. All these changes led to increased biased strengthening or weakening of certain hubs that were vital to the exchange of transactions.

This fundamental information already reveals important insights that could be used for the development of more effective and efficient training systems, by placing focus on communication and crew management under emergencies. For example, the strong contribution of cognitive influences such as memory on communication, suggests the targeting of such factors in the training process. This is echoed by BEA's recommendation FRAN-2012-042 “(...) to develop and maintain a capacity to manage crew resources when faced with the surprise generated by unexpected situations" (BEA 2012, p. 209).

Despite these initial pieces of information, of particular interest are also the exact alterations within each of the five communication dynamics, as for example, the changes of each participation shift. Specifically, examining changes in particular p-shifts could be valuable for future pilot emergency training, since they provide a clear understanding of the local rule patterns that dominate an emergency phase. For example, looking at the results of this analysis, we see that the unconscious tendency of "handing-off communication" from agent A to agent B and from B to Y (PSAB$B Y)$ is much more prominent in unexpected situations. This information could suggest the benefit of introducing a trained mediator (either human or non-human) to assist in restoration of prior successive patterns by re-directing communication; thus blocking imminent critical transitions that lead to decline in resilience. Great attention should be given to the fact that handing-off communication, as other p-shifts, reflects uninformed auto-correlation patterns, meaning that pilots were not consciously directing communication to others in a pre-meditated manner, leading to 'de-structuring of crew cooperation' and a 'total loss of cognitive control of the situation' (BEA 2012, p. 199). We thus do not claim that handing-off communication leads to malfunctioning under well-informed conditions, but rather that it is troubling in its unconscious nature. Again, this is reflected in BEA's conclusion that "the two co-pilots failed to communicate, in a clear and precise manner, the intentions and objectives that motivated the tasks they performed" (BEA 2012, p. 184).

Note that all transactions mentioned here, are not considered to be generating a Distributed Situation Awareness (DSA) network. On a transaction level of analysis, one should focus only on the architectural patterns of the network and not consider macrocognitive functions, as these belong to an analysis at the knowledge level (Newell 1982). The use of Butts' relational event model (REM) made it possible to investigate differences that unfold in the dynamic architecture patterns between a normal and unexpected situation without the need to consider specific content details. This is an important difference, as it also makes it easier for the current analysis to expand to longer time periods, and also enables the comparison of this system's network patterns to that of others. However, feeding back to a content-specific manner of the knowledge level can always provide further insight into the architectural patterns of the system, and thus the mapping of macrocognitive functions onto such patterns can prove useful. For example, in addition to the quantitative approach adopted here, and as suggested in research of macrocognitive functions, the development of aviation support and training systems can be further informed by the investigation of the macrocognitive sense-making and re-framing processes of the actors in the cockpit (Malakis and Kontogiannis 2014; Rankin et al. 2016).

This article has provided a prominent example of how a transaction level analysis can be carried out regardless of the units of analysis involved. However, the research did not involve an investigation of the entire system's network, as it focused on just the human actors at play, with none of the other non-human nodes present. Moreover, communication dynamics were tested between three agents for a limited time span, while for a considerable amount of the time analysed only two agents were present, which may have affected the power of the communication dynamics effects that required more than two nodes. This might also be the reason why the parameters for triadic effects were minor and non-significant in the present analysis. Future research should investigate the entire network in the cockpit to conclude whether triadic effects play a role in the communication dynamics and whether they change according to the situation.

The results obtained may also have been limited by the tendency of the BIC statistic to favour smaller models (Wasserman and Garry 2005). Hence, it is likely that fixed effects were less favoured for including a large set of parameters, contrary to the other single-parameter effects. Future research could consider placing more attention to the Akaike information criterion (AIC) for the parameters of individual level heterogeneity, to ensure less biased model selection. In any case, the advantages of model pruning as was completed here, lie with selecting only the models that have a strong predictive value. Thus, we encourage future analyses using REM that will include more nodes, to consider even more models for other communication dynamics, and use the BIC or AIC selection criterion to easily select and interpret only those with important information. For example, given that more nodes are considered in an analysis, such models could include investigation of other participation shifts like turn usurping (PS-ABXY) or turn claiming (PS-A0XY; Gibson 2003). We also suggest the use of the informR package (Marcum and Butts 2015) for cases of rather complex sociotechnical systems. This package allows the creation of models with more complex relational event sequences that, however, move beyond the scope of the current article. 
Despite its limitations, the current research has implications on future investigations, as it shows an innovative way of carrying out research for complex sociotechnical systems, by offering a 'global' approach to their investigation; an approach that does not require extensive background knowledge of a system, as required by other approaches, while it facilitates quantitative comparison to other sociotechnical systems. For instance, using REM, patterned changes in different systems could be compared quantitatively with respect to similarities and differences in communication patterns across different settings, and at different points in time. Also, the analysis in the present article focuses on a flight duration of $2 \mathrm{~h}$, and divided phases into only normal or emergency. However, one could use REM to study emergencies under prolonged time scales or even break down an event to more subphases in time, in that way allowing more thorough comparison to other systems. This could be valuable for the development of effective training procedures, tailored to the similar structural needs of aviation networks, or other sociotechnical systems.

An example of a comparison amongst different systems is briefly presented here, using the analysis of the communication networks in the World Trade Centre during the emergency situation on 9/11 (Butts 2008); an analysis also performed using REM. Even though Butts' analysis included investigation of only the emergency phase, similar patterns may be observed in the communication dynamics during the World Trade Centre emergency and the emergency of AF447, namely in p-shifts, reciprocity, and persistence; all of which were encouraged during the unexpected situation of both accidents. This correlation is a first indication on how an analysis at the transaction level can also be used to investigate similarities in system failures across different settings, and at different points in time.
The current analysis uses the transaction level approach to investigate patterned changes in communication dynamics under unexpected situations. This approach surpasses the limits to generalization that accompany other system levels, since it is independent of the context in which the system functions, and offers the opportunity to consider longitudinal data in the system's analysis. It can also be tailored to any specific case, by feeding back to the knowledge level. A very interesting opportunity for future investigations could be examining whether the patterns of change in the communication dynamics in a system are similar across different accidents in aviation, as initiated in this discussion, or even in other settings where complex systems have lacked graceful extensibility.

Acknowledgements The authors would like to acknowledge Iris ten Klooster for her contribution to the data analysis.

Open Access This article is distributed under the terms of the Creative Commons Attribution 4.0 International License (http://creativeco mmons.org/licenses/by/4.0/), which permits unrestricted use, distribution, and reproduction in any medium, provided you give appropriate credit to the original author(s) and the source, provide a link to the Creative Commons license, and indicate if changes were made.

\section{Appendix A}

Statistics used to assess effects, and their respective codes (Butts 2008):

Fixed effects: FESnd, FERec

Preferential attachment: NTDegRec

Triadic effects: OTPSnd, ITPSnd, OSPSnd, ISPSnd

Recency: RSndSnd

Persistence: FrPSndSnd

Participation shifts: PSAB-BA, PSAB-AY, PSAB-BY, PSAB-XB, PSAB-XA

For more details, see package relevent (Butts 2008). 


\section{Appendix B}

Table with parameter estimates for relational event models, by phase

\begin{tabular}{llllllllll}
\hline \multicolumn{7}{c}{ Normal phase } & & & Emergency phase \\
\hline & Estimate & Std error & $z$ value & $\operatorname{Pr}(>|z|)$ & & Estimate & Std error & $z$ value & Pr $>|z|)$ \\
PSAB-BA & 2.275 & 0.129 & 17.635 & $<2.2 \mathrm{e}-16^{* * *}$ & PSAB-BA & 4.223 & 0.155 & 27.313 & $<2.2 \mathrm{e}-16^{* * *}$ \\
PSAB-AY & -2.872 & -0.581 & 4.946 & $7.569 \mathrm{e}-07^{* * *}$ & PSAB-AY & 3.203 & 0.191 & 16.754 & $<2.2 \mathrm{e}-16^{* * *}$ \\
PSAB-BY & -3.281 & 0.710 & -4.623 & $3.784 \mathrm{e}-06^{* * *}$ & PSAB-BY & 1.098 & 0.454 & 2.420 & $0.01554^{*}$ \\
PSAB-XB & -3.977 & 1.002 & -3.971 & $7.154 \mathrm{e}-05^{* * *}$ & PSAB-XB & 2.395 & 0.255 & 9.382 & $<2.2 \mathrm{e}-16^{* * *}$ \\
PSAB-XA & -2.353 & 0.451 & -5.213 & $1.859 \mathrm{e}-07^{* * *}$ & PSAB-XA & 2.182 & 0.279 & 7.830 & $4.885 \mathrm{e}-15^{* * *}$ \\
T-OTP & 0.429 & 0.325 & 1.319 & 0.18703 & OTPSnd & -0.145 & 0.112 & -1.289 & 0.1974895 \\
T-ITP & 0.537 & 0.221 & 2.428 & $0.01517^{*}$ & ITPSnd & -0.186 & 0.125 & -1.489 & 0.1365508 \\
T-OSP & 0.033 & 0.0843 & 0.392 & 0.69511 & OSPSnd & -0.003 & 0.013 & -0.207 & 0.8358699 \\
T-ISP & -0.859 & 0.343 & -2.505 & $0.01224^{*}$ & ISPSnd & 0.605 & 0.172 & 3.527 & $0.0004204^{* * *}$ \\
R & 3.911 & 0.324 & 12.090 & $<2.2 \mathrm{e}-16^{* * *}$ & R & 5.230 & 0.199 & 26.325 & $<2.2 \mathrm{e}-16^{* * *}$ \\
P & 3.199 & 0.251 & 12.749 & $<2.2 \mathrm{e}-16^{* * *}$ & P & 8.477 & 0.246 & 26.698 & $<2.2 \mathrm{e}-16^{* * *}$ \\
PA & 3.197 & 0.424 & 7.533 & $4.974 \mathrm{e}-14^{* * *}$ & PA & -5.388 & 0.411 & -13.123 & $<2.2 \mathrm{e}-16^{* * *}$ \\
FESnd.1 & 0.635 & 51.450 & 0.012 & 0.9902 & FESnd.2 & 0.495 & 51.532 & 0.010 & 0.9923 \\
FESnd.2 & -0.784 & 51.478 & -0.015 & 0.9878 & FESnd.3 & 0.387 & 51.669 & 0.008 & 0.9940 \\
FERec.1 & 0.827 & 51.450 & 0.016 & 0.9872 & FERec.2 & 0.525 & 51.532 & 0.010 & 0.9919 \\
FERec.2 & -0.272 & 51.477 & -0.005 & 0.9958 & FERec.3 & 0.323 & 51.668 & 0.006 & 0.9950 \\
FEInt.1 & 1.686 & 51.451 & 0.033 & 0.9739 & FEInt.2 & 1.353 & 51.532 & 0.026 & 0.9791 \\
FEInt.2 & -1.090 & 51.477 & -0.021 & 0.9831 & FEInt.3 & 0.708 & 51.668 & 0.014 & 0.9891 \\
\hline
\end{tabular}

$* p<0.05, * * p<0.01, * * * p<0.001$

\section{References}

Alba RD (1982) Taking stock of network analysis: a decade's results. In: Bacharach SB (ed) Research in the sociology of organizations. JAI Press, Greenwich, pp 39-74

Alderson DL, Doyle JC (2010) Contrasting views of complexity and their implications for network-centric infrastructures. IEEE Trans Syst Man Cybern. https://doi.org/10.1109/tsmca.2010.2048027

Allison CK, Revell KM, Sears R, Stanton NA (2017) Systems theoretic accident model and process (STAMP) safety modelling applied to an aircraft rapid decompression event. Saf Sci 98:159-166

Amalberti R (2001) The paradoxes of almost totally safe transportation systems. Saf Sci 37:109-126

Anderson JR (2007) How can the mind occur in the physical universe? Oxford University Press, New York

Berlingerio M, Coscia M, Giannotti F, Monreale A, Pedreschi D (2011) The pursuit of hubbiness: analysis of hubs in large multidimensional networks. J Comput Sci. https://doi.org/10.1016/j. jocs.2011.05.009

Bureau d'Enquêtes et d'Analyses Pour la s Ecurit e de l'Aviation Civile (2012) Final report on the accident on 1st June 2009 to the Airbus A330-203 registered F-GZCP operated by Air France flight AF 447 Rio de Janeiro Paris. http://www.bea.aero/docspa/ 2009/fcp090601.en/pdf/f-cp090601.en.pdf. Accessed Oct 2017

Butts CT (2008) A relational event framework for social action. Sociol Methodol 38:155-200
Cacciabue PC, Hollnagel E (1995) Simulation of cognition: applications. In: Hoc JM, Cacciabue PC, Hollnagel E (eds) Expertise and technology: cognition and human-computer cooperation. Lawrence Erlbaum Associates, Hillsdale, pp 55-73

Cooke NJ, Gorman JC, Myers CW, Duran JL (2013) Interactive team cognition. Cogn Sci 37:255-285

Doyle JC, Csete ME (2011) Architecture, constraints, and behavior. Proc Natl Acad Sci USA 108(Suppl. 3):15624-15630

Farjoun M, Starbuck WH (2007) Organizing at and beyond the limits. Organ Stud. https://doi.org/10.1177/0170840607076584

Freeman LC, Romney AK, Freeman SC (1987) Cognitive structure and informant accuracy. Am Anthropol. https://doi.org/10.1525/ aa.1987.89.2.02a00020

Gibson DR (2003) Participation shifts: order and differentiation in group conversation. Soc Forces. https://doi.org/10.1353/ sof.2003.0055

Griffin TGC, Young MS, Stanton NA (2010) Investigating accident causation through information network modelling. Ergonomics 53(2): $198-210$

Hackman JR (2003) Learning more by crossing levels: evidence from airplanes, hospitals, and orchestras. J Organ Behav 24:905-922

Hoffman RR, McNeese MD (2009) A history for macrocognition. J Cogn Eng Decis Mak. https://doi.org/10.1518/155534309X441835

Hoffman RR, Woods DD (2011) Beyond Simon's slice: five fundamental trade-offs that bound the performance of macrocognitive work systems. IEEE Intell Syst. https://doi.org/10.1109/MIS.2011.97

Hollnagel E (2012) FRAM: the functional resonance analysis method. Ashagate, Farnham 
Johansson B, Hollnagel E (2007) Pre-requisites for large scale coordination. Cogn Technol Work. https://doi.org/10.1007/s1011 1-006-0050-z

Kahneman D, Klein G (2009) Conditions for intuitive expertise: a failure to disagree. Am Psychol. https://doi.org/10.1037/a0016755

Karsh B-T, Waterson P, Holden RJ (2014) Crossing levels in systems ergonomics: a framework to support 'mesoergonomic inquiry'. Appl Ergon 45(1):45-54

Klein G, Ross KG, Moon BM, Klein DE, Hoffman RR, Hollnagel E (2003) Macrocognition. IEEE Intell Syst 18:81-85

Leenders RT, Contractor NS, Dechurch LA (2016) Once upon a time. Organ Psychol Rev. https://doi.org/10.1177/2041386615578312

Leveson NG (2004) A new accident model for engineering safer systems. Saf Sci 42(4):237-270

Malakis S, Kontogiannis T (2014) Exploring team sensemaking in air traffic control (ATC): insights from a field study in low visibility operations. Cogn Technol Work. https://doi.org/10.1007/s1011 1-013-0258-7

Marcum CS, Butts CT (2015) Constructing and modifying sequence statistics for relevent using informR in R. J Stat Softw 64:1-36

Neville T, Salmon PM, Read GJM, Kalloniatas A (2016) Play on or call a foul? Testing and extending distributed situation awareness theory through sports officiating. Theor Issues Ergon Sci. https:// doi.org/10.1080/1463922X.2015.1106617

Newell A (1982) The knowledge level. Artif Intell 18:87-127

Newell A (1990) Unified theories of cognition. Harvard University Press, Cambridge

R Core Team (2013) R: a language and environment for statistical computing. R Foundation for Statistical Computing, Vienna. http:// www.R-project.org/

Rankin A, Woltjer R, Field J (2016) Sensemaking following surprise in the cockpit-a re-framing problem. Cogn Technol Work. https ://doi.org/10.1007/s10111-016-0390-2

Reason J (2000) Safety paradoxes and safety culture. Injury Control Saf Promot 7:3-14

Romney A, Faust K (1982) Predicting the structure of a communications network from recalled data. Soc Netw. https://doi. org/10.1016/0378-8733(82)90015

Roth WM (2018) Autopsy of an airplane crash: a transactional approach to forensic cognitive science. Cogn Technol Work 20:267-287

Roth WM, Jornet AG (2013) Situated cognition. WIREs Cogn Sci 4(5):463-478

Salmon PM, Walker GH, Stanton NA (2016) Pilot error versus sociotechnical systems failure: A distributed situation awareness analysis of Air France 447. Theor Issues Ergon Sci. https://doi. org/10.1080/1463922x.2015.1106618

Scheffer M (2009) Critical transitions in nature and society. Princeton University Press, Princeton

Schraagen JM (2017) Sustained adaptability: the transaction level. In: 7th REA symposium, Liège
Schraagen JMC, Klein G, Hoffman RR (2008) The macrocognition framework of naturalistic decision making. In: Schraagen JM, Militello LG, Ormerod T, Lipshitz R (eds) Naturalistic decision making and macrocognition. Ashgate Publishing Limited, Aldershot, pp 3-25

Snijders TA, Pattison PE, Robins GL, Handcock MS (2006) New specifications for exponential random graph models. Sociol Methodol. https://doi.org/10.1111/j.1467-9531.2006.00176.x

Sorensen LJ, Stanton NA (2015) Exploring compatible and incompatible transactions in teams. Cogn Technol Work 17:367-380

Stanton NA, Harvey C (2017) Beyond human error taxonomies in assessment of risk in sociotechnical systems: a new paradigm with the EAST 'broken-links' approach. Ergonomics 60(2):221-233

Stanton NA, Salmon PM, Walker GH, Baber C, Jenkins D (2005) Human factors methods: a practical guide for engineering and design, 1st edn. Ashgate, Aldershot

Stanton NA, Stewart R, Harris D, Houghton RJ, Baber C, McMaster R, Salmon PM et al (2006) Distributed situation awareness in dynamic systems: theoretical development and application of an ergonomics methodology. Ergonomics. https://doi. org/10.1080/00140130600612762

Stanton NA, Baber C, Harris D (2008) Modelling command and control: event analysis of systematic teamwork. Ashgate, Surrey

Stanton NA, Salmon PM, Walker GH, Jenkins DP (2009) Genotype and phenotype schema and their role in distributed situation awareness in collaborative systems. Theor Issues Ergon Sci 10:43-68

Stanton NA, Salmon PM, Rafferty L, Walker G, Baber C, Jenkins DP (2013) Human factors methods: a practical guide for engineering and design, 2nd edn. Ashgate, Aldershot

Stanton NA, Harris D, Starr A (2016) The future flight deck: modelling dual, single and distributed crewing options. Appl Ergon 53:331-342

Vicente KJ (1999) Cognitive work analysis: toward safe, productive, and healthy computer-based work. Lawrence Erlbaum Associates, Mahwah

Wasserman S, Faust K (1994) Social network analysis: methods and applications. Cambridge University Press, New York

Wasserman S, Garry R (2005) An introduction to random graphs, dependence graphs, and $p^{*}$. In: Carrington PJ, Scott J, Wasserman S (eds) Models and methods in social network analysis. Cambridge University Press, Cambridge, pp 192-214

Wickham H (2009) ggplot2: elegant graphics for data analysis. Springer, New York

Woods DD (2015) Four concepts for resilience and the implications for the future of resilience engineering. Reliab Eng Syst Saf. https:// doi.org/10.1016/j.ress.2015.03.018

Woods DD (2016) The risks of autonomy: Doyle's catch. J Cogn Eng Decis Mak 10(2):131-133 